\title{
Variabilidad interanual de las precipitaciones en la Región Serrana de Rio de Janeiro, en el período 1971-2010
}

jaenio Ferreira Silva Junior ${ }^{1}$ jaeniojunior@hotmail.com, Rosemary Vieira ${ }^{1}$ rosemaryvieira@id.uff.br

\begin{abstract}
RESUMEN
Se presentan datos de precipitación de tres ciudades de la Región Serrana de Rio de Janeiro: Nova Friburgo, Petrópolis e Teresópolis, para el período 1971-2010. Con el objetivo de identificar los meses y años con elevados índices de precipitación en el área y analizar la variabilidad interanual de la precipitación, fueron calculados los promedios mensuales y anuales, el total de precipitación para cada año en el área, y los valores máximos de precipitación anual. Se observó que las tres ciudades más importantes de la Región Serrana han presentado lluvias más intensas y asociadas a la acción de la Zona de Convergencia del Atlántico Sur, en especial, durante la década del 2000, lo que derivó en la ocurrencia de desastres naturales. A pesar de los resultados encontrados en la investigación, no es posible aún concluir que existe una tendencia de continuidad de los eventos pluviométricos analizados.

Palabras-clave: Desastres naturales, deslizamientos, precipitación, Región Serrana, Zona de Convergencia del Atlántico Sur.
\end{abstract}

\section{Precipitation analysis in the Rio de Janeiro Serrana Región during the period 1971-2010}

\begin{abstract}
This paper presents precipitation data from three cities in the Serrana region of Rio de Janeiro: Nova Friburgo, Petropolis and Teresopolis, during the period 1971-2010. Monthly and annual averages, total precipitation for each year, and maximum values of annual rainfall were calculated in order to identify the months and years with high levels of precipitation in the area and to analyze the interannual variability of precipitation. It was observed that the three most important cities in the Rio de Janeiro Serrana Region presented intense rainfall associated with the action of Atlantic Convergence South Zone, which has resulted in natural disasters. However, we can not conclude that there is a continuing trend of such events.
\end{abstract}

Key-words: Natural disasters, landslides, precipitation, Rio de Janeiro, South Atlantic Convergence Zone

Recibido el 14 de Junio de 2013, aceptado el 10 julio de 2013.

\footnotetext{
Laboratório de Processos Sedimentares e Ambientais (LAPSA)

Instituto Nacional de Ciência e Tecnologia da Criosfera

UFF - Instituto de Geociências - Depto de Geografia

Av. Gal. Milton Tavares de Souza, s/n

Campus da Praia Vermelha

Boa Viagem - Niterói/RJ.
} 


\section{INTRODUCCIÓN}

Tradicionalmente, la temperatura del aire y la precipitación son consideradas indicadores clásicos de alteraciones del clima. Sin embargo, es generalmente aceptado que los cambios en los patrones de precipitación, y los eventos extremos pueden tener fuertes impactos en el medio ambiente $\mathrm{y}$ en la sociedad, aunque las tendencias en la precipitación acumulada o en el promedio de la temperatura sean en escala anual o estacional (MARENGO et al. 2007).

El Grupo Intergubernamental de Expertos sobre el Cambio Climático (Intergovernmental Panel on Climate Change - IPCC) concluye en su Cuarto Informe (IPCC 2007) que existen evidencias de que eventos extremos como sequías, crecidas fluviales, ondas de calor y de frío, $\mathrm{y}$ tormentas se han intensificado en los últimos años afectando partes distintas del planeta y produciendo grandes pérdidas económicas y de vidas. Diversos estudios científicos proyectan tendencias de aumento de la ocurrencia y de la intensidad de eventos climáticos extremos en todo el mundo, aunque no se pueda correlacionar directamente a los cambios climáticos, una vez que ello son un aspecto integral de la variabilidad climática (GROISMAN et al. 2005; MARENGO 2009). En los últimos años, las Ciencias Climáticas han producido importantes avances en la investigación, tendiente a mejorar las proyecciones de los eventos climáticos más severos (LIMA 2010).

Evaluaciones del IPCC (2007) han mostrado que eventos extremos de precipitación pueden aumentar aún más que el promedio, lo que puede implicar crecidas e inundaciones más severas e intensas en un clima más cálido. TRENBERTH (2005) sugirió que la intensificación en los extremos de precipitación puede ser causada por el aumento del contenido de humedad de la atmósfera, lo que puede incrementar la disponibilidad de humedad para los sistemas atmosféricos, tales como frentes, tempestades tropicales y extratropicales y Complejos Convectivos de Mesoscala (CCM). La sucesión de eventos meteorológicos extremos ha impactado toda la comunidad global, siendo causa principal de los desastres naturales asociados (LIMA 2010).

Los eventos denominados desastres naturales que ocurren en Brasil son en su mayoría, de origen atmosférico. Entre esos, se destacan las inundaciones, crecidas bruscas, deslizamientos y sequías que están directamente relacionadas con el exceso o ausencia de lluvia (MARENGO 2009). En el Sudeste de Brasil (SEB), durante el verano es frecuente la ocurrencia de lluvias intensas que generan gran impacto en la población.

Según las Naciones Unidas, una de las más impresionantes catástrofes climáticas en el mundo se dio en la Región Serrana de Rio de Janeiro en 11 y 12 de Enero de 2011. La Región Serrana (Fig. 1) fue devastada bruscamente por lluvias intensas, con deslizamientos en las vertientes, generando pérdidas en miles de personas, junto con los daños ambientales, sociales y económicos asociados.

ApartirdelosinformesdelDEPARTAMENTO DE RECURSOS MINERALES (2010a, 2010b, 2011), solamente en el Estado de Rio de Janeiro, entre Diciembre de 2009 y Enero de 2011, más de 1.000 personas perdieron sus vidas en eventos extremos de precipitación: ciudad de Angra dos Reis (52 muertos en Enero de 2010; ciudad de Niterói (166 muertos en Abril de 2010); Región Serrana (947 muertos y 653 desaparecidos en Enero de 2011). Tras dos años del desastre, 165 personas siguen desaparecidas, según el Programa de Localização e Identificação de Desaparecidos (PLID), del Ministério Público do Rio de Janeiro (http://plid.mp.rj. gov.br/2013). 


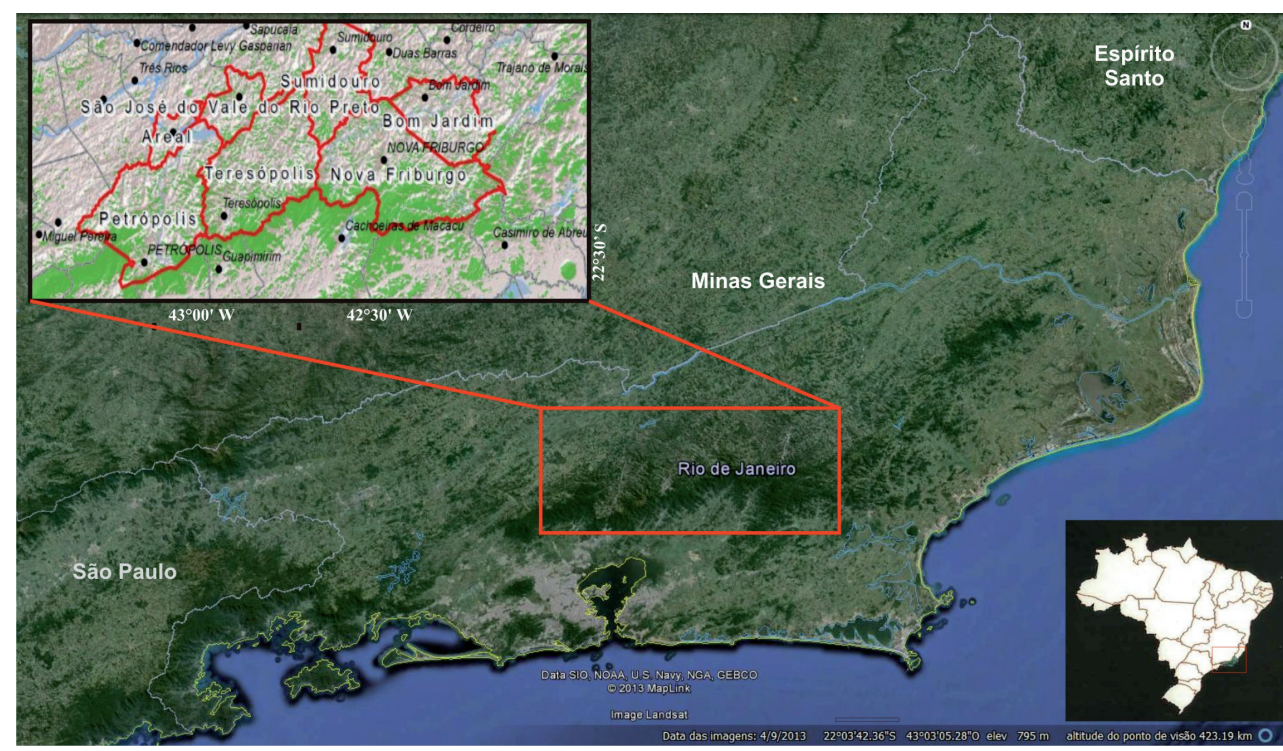

Fig. 1. Región Serrana del Estado de Rio de Janeiro.

Fig. 1. Serrana region of Rio de Janeiro State.

La Región Serrana de Rio de Janeiro es parte del gran sistema de escarpas montañosas, colinas y valles conocido como Serra do Mar, con cerca de $1.000 \mathrm{~km}$ de extensión (SANTOS 2004), extendiéndose por la costa de los Estados de Rio de Janeiro, São Paulo, Paraná y Santa Catarina. Las más altas cumbres de la Serra do Mar, con altitudes superiores a 2.300 m.s.n.m., están en la Región Serrana del Estado de Rio de Janeiro, más precisamente en las ciudades de Nova Friburgo, Teresópolis y Petrópolis. La topografía, la configuración y la localización de la Serra do Mar próximo de la costa (ALMEIDA \& CARNEIRO 1998; SANTOS 2004, 2008; SELUCH \& CHOU 2009) son elementos geográficos importantes para la comprensión de las características climáticas de la región. La combinación de elevada humedad en las capas más bajas de la atmósfera, lo que es típico en la región costera, con el aire cálido de verano y su ascensión por las montañas origina condiciones termodinámicas inestables en casi todos los días (SELUCH \& CHOU 2009). Además, la región es afectada por sistemas de larga escala e inestabilidad convectiva, generando precipitaciones fuertes y constantes.

La disposición actual de la Serra do Mar está relacionada a procesos de erosión, deslizamientos de vertientes, flujos de barro $\mathrm{y}$ de detritos, los cuales originaron nuevas áreas, algunas con topografía suave. Debido a esos procesos a lo largo de millones de años, la Serra do Mar presenta vertientes naturalmente inestables y muy susceptibles a la ocurrencia de deslizamientos, como en todas las regiones tropicales húmedas del planeta (SANTOS 2004, 2008). La figura 2 exhibe como ejemplo el área de la carretera que une las ciudades de Nova Friburgo y Teresópolis, uno de los sectores más impactados por las fuertes lluvias de enero de 2011. Las escarpas del área son formadas por rocas graníticas y gnéisicas y, en general, presentan suelos pocos espesos y bastante lixiviados (DANTAS et al. 2005).

La elevada concentración demográfica: en Petrópolis $372 \mathrm{hab} / \mathrm{km}^{2}$, en Nova Friburgo $195 \mathrm{hab} / \mathrm{km}^{2}$ y en Teresópolis $212 \mathrm{hab} / \mathrm{km}^{2}$ (InstitutoBrasileirodeGeografiaeEstatística 
http://www.ibge.gov.br/cidadesat/index. php) y la ocupación poblacional de los valles y vertientes empinadas de la Serra do Mar, con habitaciones precarias e irregulares, le imprimen a esta región una condición de susceptibilidad a la ocurrencia de desastres naturales. Según el Instituto Brasileiro de Geografia e Estatística (http://www.ibge.gov.br/cidadesat/index. php), las tres ciudades más importantes de la Región Serrana (Petrópolis, Nova Friburgo y Teresópolis), en 2010, tenían $295.917,182.082$ y 163.746 habitantes, respectivamente. De las 12 ciudades que son parte de la Región Serrana de Rio de Janeiro, 6 son consideradas como ciudades con situaciones de riesgo alto y muy alto frente a las inundaciones, crecidas y deslizamientos (SANTOS 2012).

Registros históricos revelan la ocurrencia de deslizamientos e inundaciones a lo largo de la Serra do Mar, en la Región Sudeste, asociados a elevadas precipitaciones desde el siglo XX hasta los años más recientes, en el siglo XXI. Algunos casos históricos incluyen las ciudades de Santos y São Paulo en 1928, 1947 y 1956; en la Región Metropolitana de Rio de Janeiro en 1940, 1948, 1966 y 1967 (COELHO NETTO et al. 2012). En la últimas décadas eventos catastróficos con deslizamientos de tierra en las vertientes e inundaciones fueron registrados en la ciudad de Rio de Janeiro (1982, 1983, 1988, 1991, 1999, 2009 у 2010), en São Paulo (2009, 2010 y 2011) y en la Región Serrana de Rio de Janeiro (1978, 1981, 1982, 1983, 1987,1988, 1995, 1999, 2000, 2003, 2007, 2008 у 2011 (MINISTERIO DO MEIO AMBIENTE 2011; COELHO NETTO et al. 2012).

Los casos de precipitación intensa en el Sudeste de Brasil, en especial, en los Estados de São Paulo y Rio de Janeiro han estado relacionados con la acción de Sistemas Frontales (SF) y de la Zona de Convergencia del Atlántico Sur (SACZ-South Atlantic Convergence Zone) (VIANELLO 1991; LIMA et al. 2009; LIMA 2010), que se caracteriza por una banda de nubosidad convectiva, extendiéndose en general desde la Amazonía hasta el Océano Atlántico, en dirección NE-SE (KOUSKY 1998; CARVALHO et al. 2004). Ese sistema es típico de los meses de verano, durante el monzón sur americano de verano (GAN et al. 2009), cuando la convección tropical más acentuada contribuye para la generación y mantención del fenómeno (KODAMA, 1992). La mayoría de los casos de precipitación extrema se registran entre octubre y marzo y es en esa época que se producen los eventos por la acción de la SACZ, la cual permanece por varios días sobre el Sudeste de Brasil (CARVALHO et al. 2002). Esos dos sistemas difieren en un aspecto importante, su duración. Los Sistemas Frontales impactan la región por uno o dos días, mientras la SACZ que es un sistema semi-estacionario permanece sobre el SEB por más de tres días, en general (NUNES et al. 2007; LIMA et al. 2010)

La influencia de eventos El Niño igualmente es notada respecto a los eventos intensos de precipitación (GRIMM 2003; GRIMM \& TEDESCHI 2009). Durante el mes de noviembre, en un inicio de año El Niño, hay registro de más casos extremos de precipitación en el Sur de Brasil y menos casos en la Región Centro-Este (que incluye gran parte del SEB). En el mes de enero, ese patrón se altera, propiciando más casos de precipitación intensa en la Región CentroEste. Lo mismo ocurre en los casos de eventos La Niña, pero con señales opuestas. Existe también una elevada correlación de casos extremos de precipitación, con la Temperatura Superficial del Mar (TSM) del Océano Pacífico Central y Este, la cual fue detectada por LIEBMANN et al. (2001) durante el periodo de octubre a marzo.

El propósito de esta investigación es analizar los eventos de precipitación en la Región Serrana del Estado de Rio de Janeiro en los últimos 40 años, así como sus fluctuaciones espaciales y temporales, y buscar tendencias de ocurrencia de eventos extremos. 


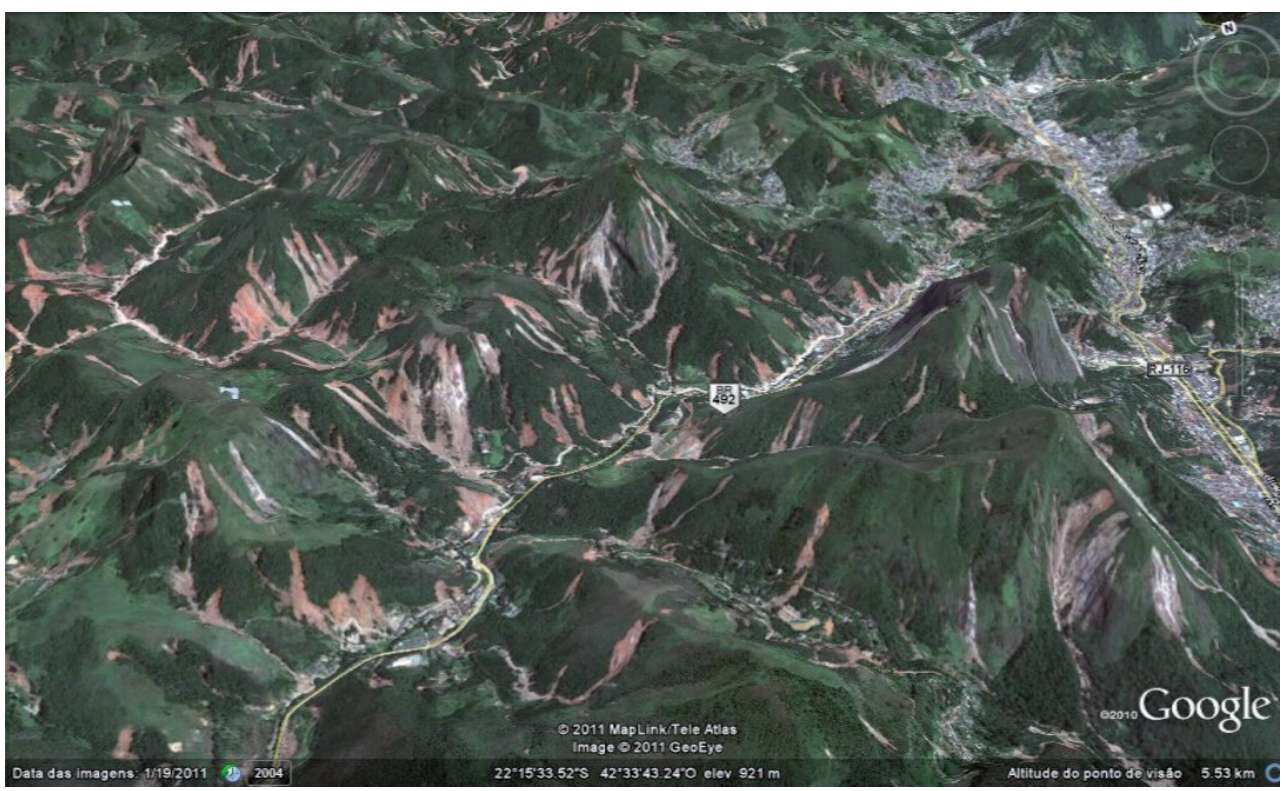

Fig. 2. Sector de la carretera que une las ciudades de Nova Friburgo y Teresópolis, impactado intensamente por el desastre de enero de 2011. En la zona central y superior derecha de la imagen se observa el centro de la ciudad de Nova Friburgo, que ocupa el valle angosto y sobre las vertientes.

Fig. 2. Road sector between the cities of Nova Friburgo and Teresópolis, which was strongly affected by the disaster in January 2011. The downtown of Nova Friburgo is seen at the central and top right corner. The city spreads out across the narrow valley end over the slopes.

\section{MATERIALES Y MÉTODOS}

Se emplearon datos de precipitación provenientes de las estaciones pluviométricas administradas por la Agência Nacional de Águas (ANA), que fueron obtenidos directamente del sitio http://hidroweb.ana.gov.br.

Los estudios y antecedentes relacionados a eventos de precipitación extrema son limitados y de difícil acceso, sobre todo, en relación a las dificultades de adquisición de series temporales climáticas prolongadas y homogéneas

Fueron reunidos datos de todas las estaciones pluviométricas disponibles para las ciudades de Nova Friburgo, Petrópolis, Teresópolis, Bom Jardim y Sumidouro. Se realizó un análisis en cada estación para seleccionar las que disponían de una serie histórica de a lo menos 30 años y en casos de ausencia de secuencia de datos, esa sería menos de tres años. Los datos presentados en este trabajo corresponden a la serie histórica de 1971-2010 de las estaciones de Nova Friburgo, Petrópolis y Teresópolis.

Con el objetivo de identificar los meses y años con elevados índices de precipitación, y así realizar el análisis de la variabilidad interanual, se calcularon los promedios mensuales y anuales; el total de precipitación para cada año y valores máximos de precipitación total mensual y anual. Como las lluvias en la región son más intensas entre Octubre y Marzo (ALVES et al. 2005), fue elaborada una distribución del promedio mensual de la precipitación por décadas para identificar las variaciones decadales en las ciudades. 
Una vez encontradas tales alteraciones, se comparó los valores totales mensuales de las décadas identificadas con el promedio de la serie histórica, 1971-2010. Los gráficos e histogramas fueron construidos con el software Grapher.

La actuación de la SACZ, El Niño/La Niña y de los Sistemas Frontales fue adquirida en el Boletim de Informações Climáticas y en el Boletím Climanálise (período 1990-2010), del Instituto Nacional de Pesquisa Espaciais (INPE - www.inpe.br). Con respecto a la SACZ se adjunta los eventos identificados por QUADRO (1994) en el período 198089. Los años de comportamiento de El Niño/ La Niña fueron obtenidos por el sitio de la Agencia Americana de Océano y Atmósfera (NOAA): http://www.cpc.ncep.noaa.gov/ products/analysis_monitoring/ensostuff/ ensoyears.shtml,

La información sobre los desastres asociados a las lluvias notificados en las ciudades de Nova Friburgo, Petrópolis y Teresópolis fueron obtenidos de la Secretaria Nacional de Defesa Civil.

\section{RESULTADOS Y DISCUSIÓN}

La evolución de la precipitación anual y precipitación máxima anual de las ciudades de Nova Friburgo, Petrópolis y Teresópolis, correspondientes al periodo de estudio de 40 años se observa en la figura 3. La precipitación máxima anual está basada en el mes de cada año que presentó los mayores valores acumulados.

Se aprecia el elevado volumen pluviométrico en las tres ciudades destacando Nova Friburgo y Teresópolis, con valores máximos de precipitación superiores a $100 \mathrm{~mm}$ en toda la serie histórica e indicios de aumento a partir de la segunda mitad de la década de los 80. Petrópolis difiere en el volumen inferior de precipitación y en la condición de aumento de la precipitación a partir de los años 2000, aunque las décadas anteriores hayan sido lluviosas. Nova Friburgo es la ciudad que presenta volúmenes más elevados: la primera mitad de la década de 1980 estuvo caracterizada por valores elevados de precipitaciones y con máximas anuales que superaron los $800 \mathrm{~mm}$, como el año 1983. Sin embargo, a partir de fines de la década de 1980, los totales anuales superaron los $500 \mathrm{~mm}$ en casi todos los años y, en la década de 2000, sobre los $600 \mathrm{~mm}$, como en el caso de 2007, sobrepasando los $800 \mathrm{~mm}$. 

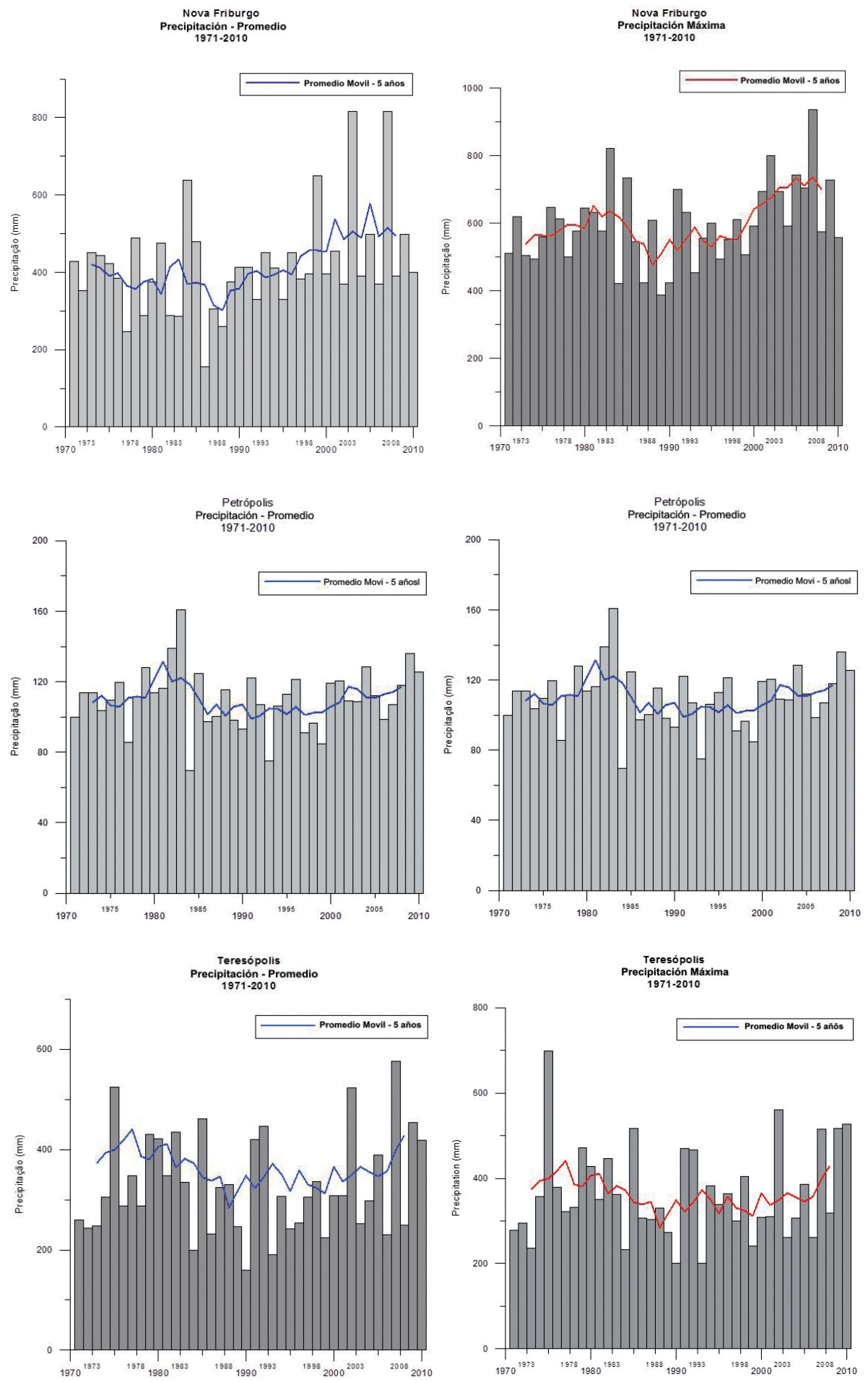

Fig. 3. Histograma de distribución de precipitación en el periodo 1971-2010 en Nova Friburgo, Petrópolis y Teresópolis: (a) Precipitación máxima; (b) Promedio.

Fig. 3. Histogram distribution of precipitation in the period 1971-2010 in Nova Friburgo, Petrópolis and Teresópolis: (a) maximum precipitation, (b) Average annual precipitation. 
Al buscar la relación entre la precipitación con los eventos El Niño/La Niña y con la acción del sistema de la Zona de Convergencia del Atlántico Sur sobre la región, se verificó, por ejemplo, en Nova Friburgo, influencias del El Niño/La NiñaOscilación Sur más significativas en las décadas de los 70 y los 80 (tres eventos en los años 70 y cinco en los años 80). Fueron identificados cinco eventos SACZ en la década de 1980. Por otro lado, la influencia de la SACZ fue más intensa en las décadas de 1990 y 2000: 10 episodios en la década de 90 y 14 en la década de 2000. Hubo 3 eventos El Niño en los años 90 y 1 en los años 2000 (Fig. 4). Los gráficos de la figura 5 exhiben los valores promedios de los totales acumulados en cada mes y los totales máximos mensuales.

Se observa también en la figura 5 la variabilidad interanual de la precipitación asociada al sistema de monzón sur americano que actúa sobre gran parte del territorio brasileño, con grandes totales mensuales de precipitación en los meses de primavera y verano, valores más bajos en el otoño e invierno (GAN et al. 2009).
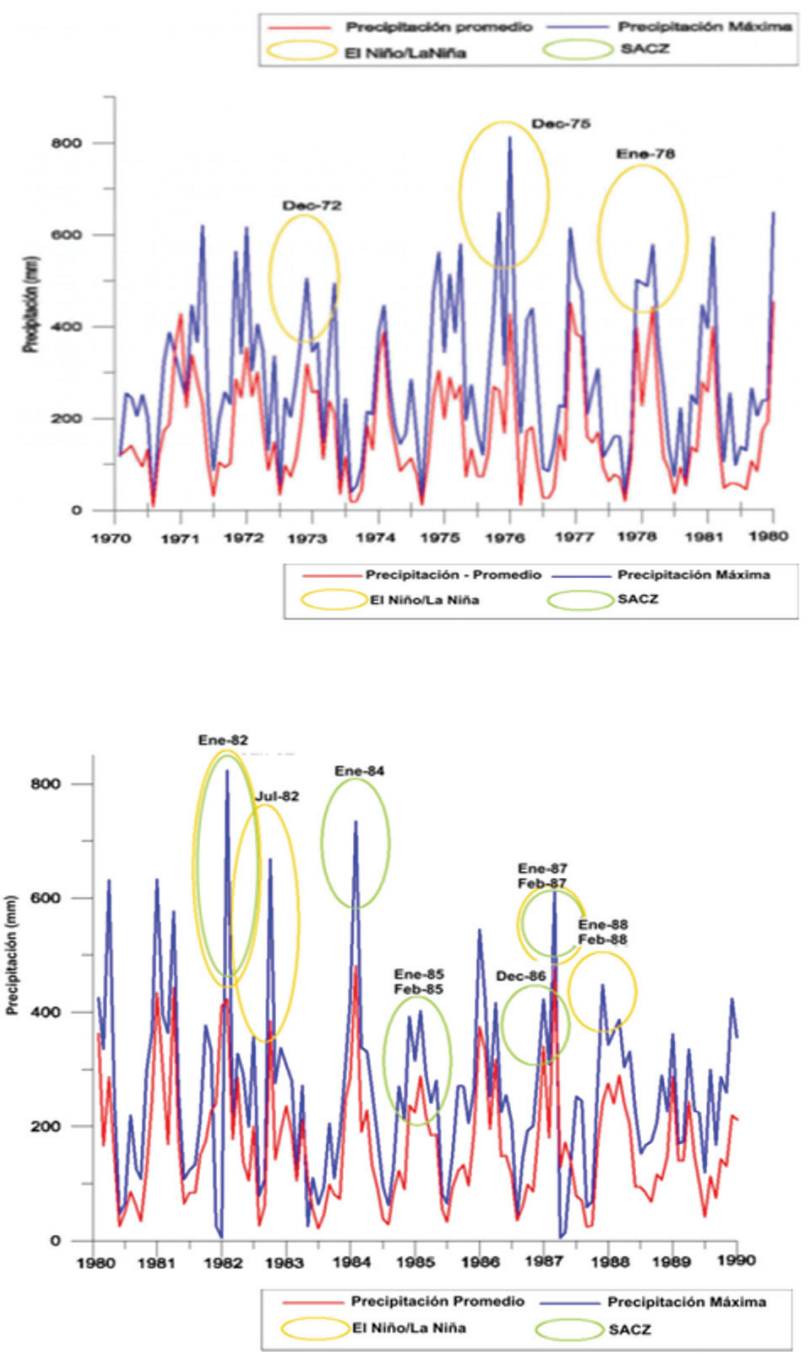

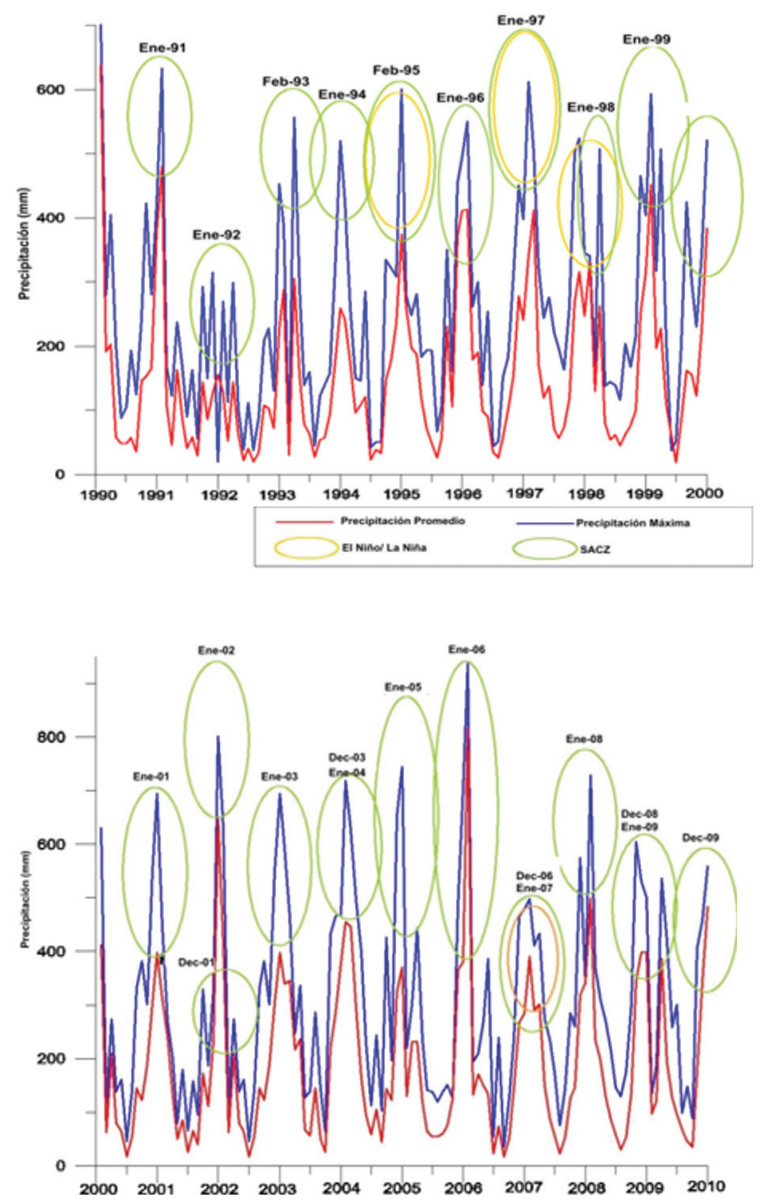

Fig. 4. Promedio de la precipitación mensual y precipitación máxima mensual distribuidas por décadas en Nova Friburgo.

Fig. 4. Average monthly rainfall and monthly maximum precipitation distributed for decades in Nova Friburgo.

Los gráficos de la figura 5 muestran la distribución del promedio mensual en el periodo Octubre-Marzo por décadas. De acuerdo con la figura 6 en las tres ciudades el promedio mensual de la precipitación fue superior en la década de 2000, ocurriendo en Nova Friburgo la década más lluviosa en el trimestre Octubre-Noviembre-Diciembre (OND) así como en el trimestre EneroFebrero-Marzo (EFM). En Petrópolis y Teresópolis, igualmente la década del 2000 fue la más lluviosa en los meses de Enero y Febrero. Esos escenarios ya se perciben desde la década del 90 para las tres ciudades.
El mes de Enero del año de 2011 siguió ese patrón de elevada precipitación generando la tragedia climática del país, que devastó las tres ciudades y otras más de la región serrana. Entre los días 11 y 12 de enero, en un período de $24 \mathrm{hrs}$, la estación del Instituto Nacional de Meteorología (INMET) registró $182,8 \mathrm{~mm}$ en Nova Friburgo. Es necesario considerar que en el día 10 de enero llovió más de $90 \mathrm{~mm}$, lo que contribuyó para la saturación del suelo y la generación de los deslizamientos en las vertientes. 

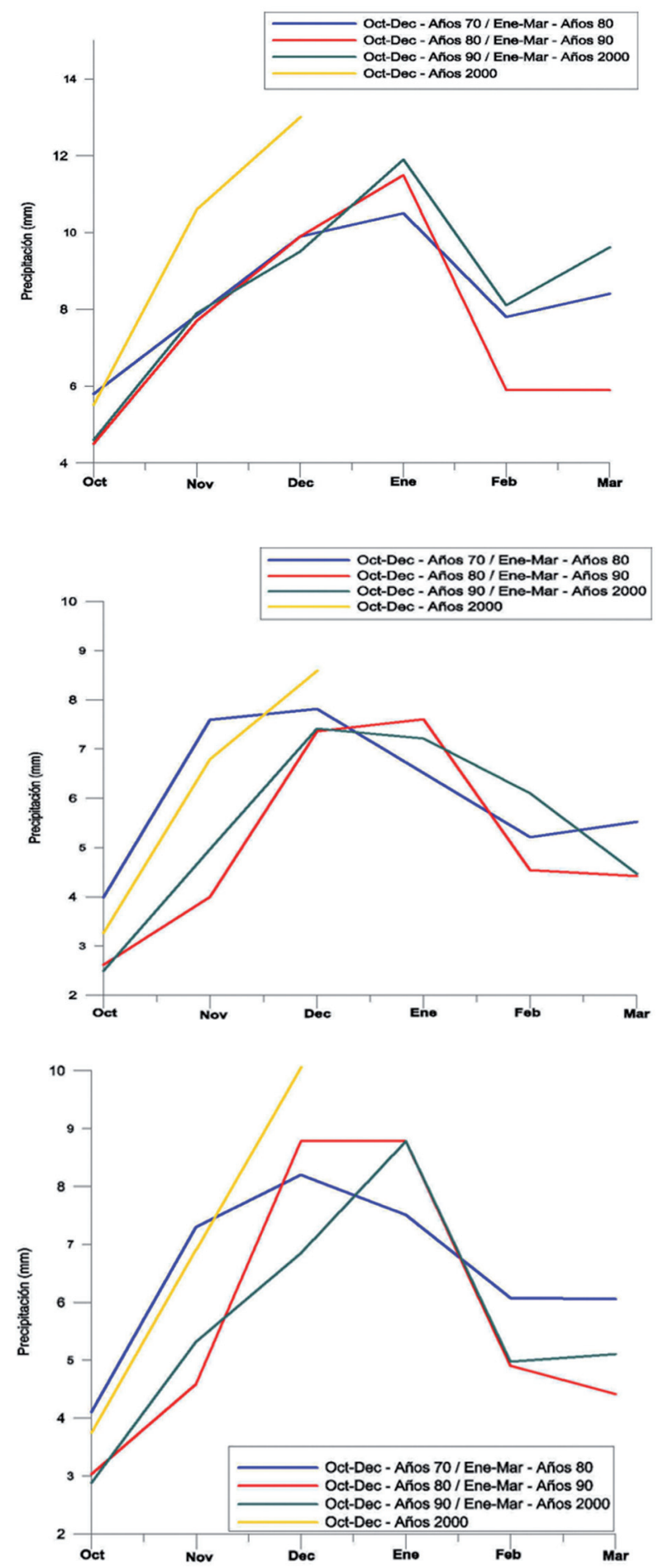

Fig. 5. Distribución por décadas del promedio mensual la precipitación en el período OctubreMarzo, en las ciudades de Nova Friburgo (a), Petrópolis (b) y Teresópolis (c).

Fig. 5. Distribution by decades of average monthly precipitation from October to March, in Nova Friburgo (a), Petrópolis (b) and Teresópolis (c). 
En la década de 2000 ocurrieron los promedios mensuales, anuales y máximos diarios de precipitación más elevados: las figuras 6,7 y 8 muestran el comportamiento de los datos en esta década en las tres ciudades, comparados al promedio de la serie histórica (1971-2010).

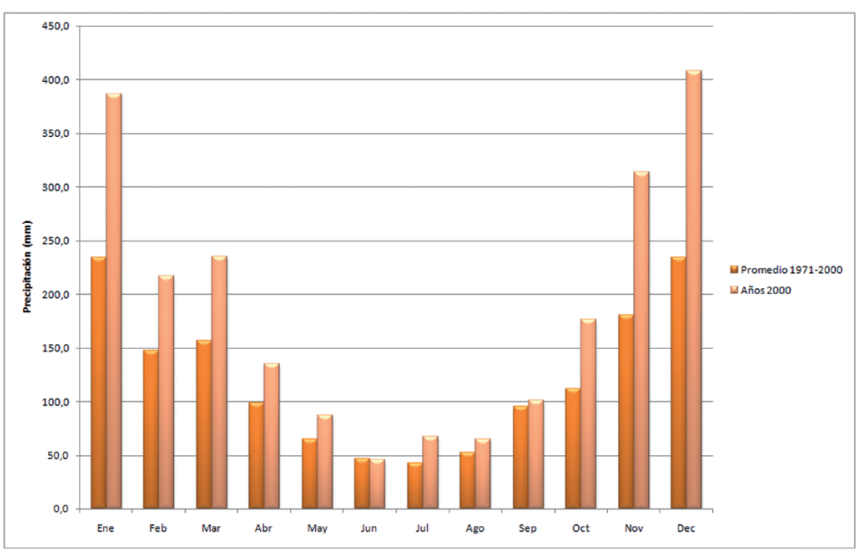

\begin{tabular}{|c|c|c|c|}
\hline Mes & $\begin{array}{c}\text { Promedio } \\
\mathbf{1 9 7 1 - 2 0 0 0} \\
(\mathbf{m m})\end{array}$ & $\begin{array}{c}\text { Total Mensual } \\
\text { Años 2000 } \\
(\mathbf{m m})\end{array}$ & $\%$ \\
\hline Ene & 234,7 & 386,1 & +65 \\
\hline Feb & 147,3 & 216,6 & +47 \\
\hline Mar & 156,9 & 234,7 & +50 \\
\hline Abr & 98,5 & 134,9 & +37 \\
\hline May & 64,7 & 87,2 & +35 \\
\hline Jun & 46,9 & 46,0 & -2 \\
\hline Jul & 42,8 & 67,2 & +57 \\
\hline Ago & 52,7 & 64,9 & +23 \\
\hline Sep & 95,0 & 100,8 & +6 \\
\hline Oct & 112,2 & 176,5 & +57 \\
\hline Nov & 180,2 & 313,9 & +74 \\
\hline Dez & 234,5 & 408,2 & +74 \\
\hline
\end{tabular}

Fig. 6. Precipitaciones totales mensuales de la década de 2000 comparados al promedio del período 1971-2000 en Nova Friburgo. En la tabla los porcentuales de precipitación sobre/abajo del promedio de 1971-2000 en Nova Friburgo.

Fig. 6. Total Monthly Precipitation in the 2000s compared to the 1971-2000 average in Nova Friburgo. In the table the percentage of precipitation above / below the 1971-2000 average in Nova Friburgo.

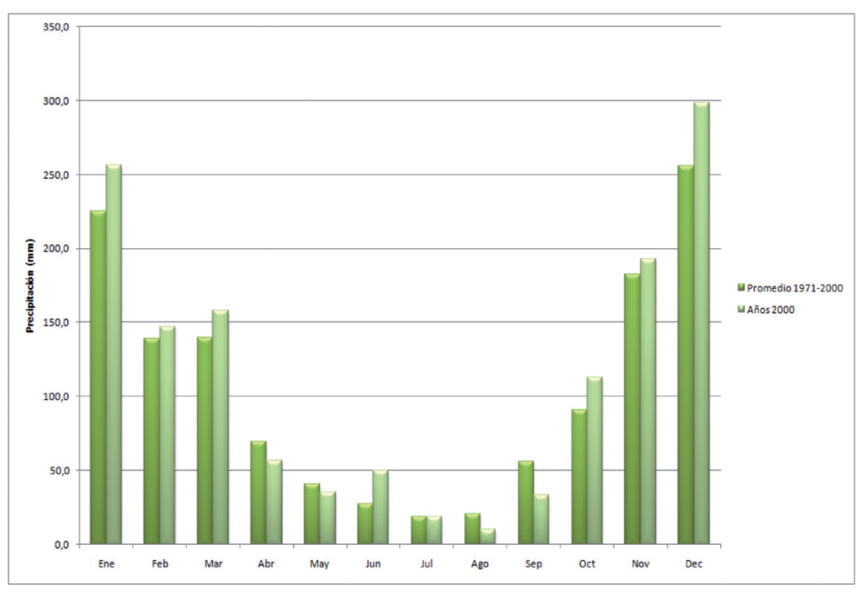

\begin{tabular}{|c|r|r|r|}
\hline Mes & $\begin{array}{r}\text { Promedio } \\
\mathbf{1 9 7 1 - 2 0 0 0} \\
(\mathbf{m m})\end{array}$ & $\begin{array}{c}\text { Total Mensual } \\
\text { Años 2000 } \\
(\mathbf{m m})\end{array}$ & \% \\
\hline Ene & 225,1 & 256,4 & +14 \\
\hline Feb & 139,1 & 146,8 & +06 \\
\hline Mar & 139,7 & 157,9 & +13 \\
\hline Abr & 69,3 & 56,4 & -19 \\
\hline May & 40,8 & 35,5 & -13 \\
\hline Jun & 27,3 & 50,1 & +84 \\
\hline Jul & 19,0 & 18,7 & -2 \\
\hline Ago & 20,3 & 10,1 & -50 \\
\hline Sep & 55,8 & 33,5 & -40 \\
\hline Oct & 91,0 & 112,8 & +24 \\
\hline Nov & 182,4 & 192,8 & +6 \\
\hline Dec & 256,0 & 298,3 & +17 \\
\hline
\end{tabular}

Fig.7. Figura 8. Precipitaciones totales mensuales de la década de 2000 comparados al promedio del período 1971-2000 en Petrópolis. En la tabla los porcentuales de precipitación sobre/abajo del promedio de 1971-2000 en Petrópolis.

Fig. 7. Total Monthly Precipitation in the 2000s compared to the 1971-2000 average in Petrópolis. In the table the percentage of precipitation above / below the 1971-2000 average in Petrópolis. 


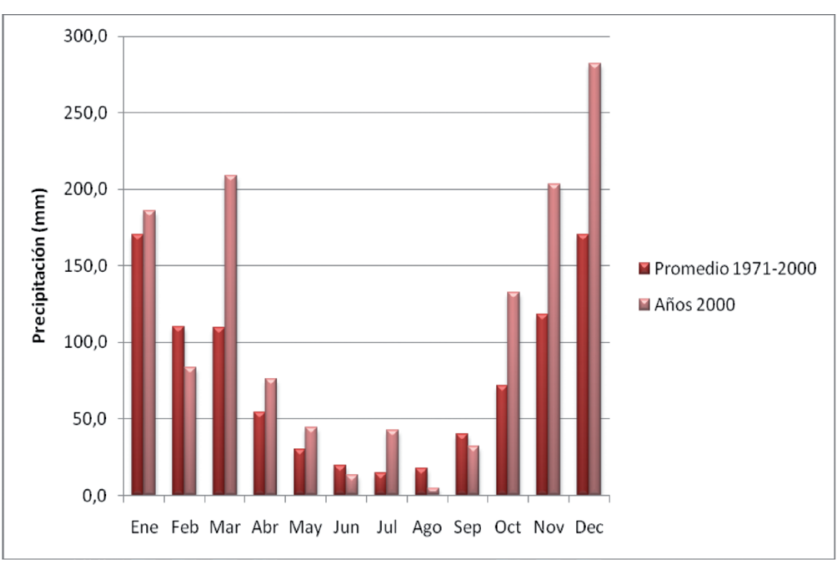

\begin{tabular}{|c|r|r|r|}
\hline Mes & $\begin{array}{c}\text { Promedio } \\
\mathbf{1 9 7 1 - 2 0 0 0} \\
(\mathbf{m m})\end{array}$ & $\begin{array}{c}\text { Total Menusal } \\
\text { Años 2000 } \\
(\mathbf{m m})\end{array}$ & \% \\
\hline Ene & 170,3 & 186,0 & +9 \\
\hline Feb & 110,2 & 83,4 & -24 \\
\hline Mar & 109,6 & 209,1 & +91 \\
\hline Abr & 54,4 & 76,0 & +40 \\
\hline May & 29,8 & 44,1 & +8 \\
\hline Jun & 19,2 & 13,0 & -32 \\
\hline Jul & 14,7 & 42,6 & +190 \\
\hline Ago & 17,5 & 4,3 & -75 \\
\hline Sep & 40,0 & 31,8 & -21 \\
\hline Oct & 71,4 & 132,7 & +86 \\
\hline Nov & 118,4 & 203 & +72 \\
\hline Dec & 170,6 & 282,2 & +65 \\
\hline
\end{tabular}

Fig. 8. Precipitaciones totales mensuales de la década de 2000 comparados al promedio del período 1971-2000 en Teresópolis. En la tabla los porcentuales de precipitación sobre/bajo del promedio de 1971-2000 en Teresópolis.

Fig. 8. Total Monthly Precipitation in the 2000s compared to the 1971-2000 average in Nova Teresópolis. In the table the percentage of precipitation above / below the 1971-2000 average in Teresópolis.

En Nova Friburgo, el mes de Enero presentó valores muy superiores al promedio, seguido de los meses de Noviembre y Diciembre. Las precipitaciones de invierno estuvieron también sobre el promedio, salvo el mes de junio.

Pese a que los totales mensuales de precipitaciones en Petrópolis son más bajos que los de Nova Friburgo, la proporción de los valores de la década del 2000 con el promedio de 1971-2010 supera la de Nova Friburgo. El trimestre OND de la década del 2000 en la ciudad de Teresópolis, presentó precipitaciones totales mensuales superiores al promedio de 1971-2000, sin observarse, sin embargo, los grandes montos registrados en Nova Friburgo. La excepción son los valores de precipitaciones totales correspondientes al mes de Diciembre y al mes de Junio. El mes de Julio presentó valores superiores en Nova Friburgo y Petrópolis no tuvo el mismo comportamiento en Teresópolis.

La Tabla 1 presenta notificaciones en la Defensa Civil de Desastres Naturales relacionados de las tres ciudades. Se observa el alto número de notificaciones de Situación de Emergencia en la ciudad de Petrópolis producidas por crecidas y deslizamientos a lo largo de los años 2000, aunque los volúmenes de precipitación máxima acumulados en $24 \mathrm{hrs}$ alcanzaron valores mayores en Nova Friburgo (Fig. 9). Es importante observar que las fechas de notificaciones en las tres ciudades coinciden con los períodos de actividad de la SACZ, lo que se infiere el rol fundamental de ese sistema en la intensificación de las precipitaciones durante el verano $\mathrm{y}$, por consecuencia, en los desastres naturales en la región serrana.

Diversos estudios han encontrado una fuerte relación entre la acción de la SACZ, lluvias intensas y la ocurrencia de desastres. CARVALHO et al. (2002) concluyó que $35 \%$ de los episodios de lluvias intensas en el Estado de São Paulo son debidos a la SACZ. LIMA et al. (2010) verificó que 47\% de los casos en todo el SEB son resultados de la acción de la SACZ. 
a)

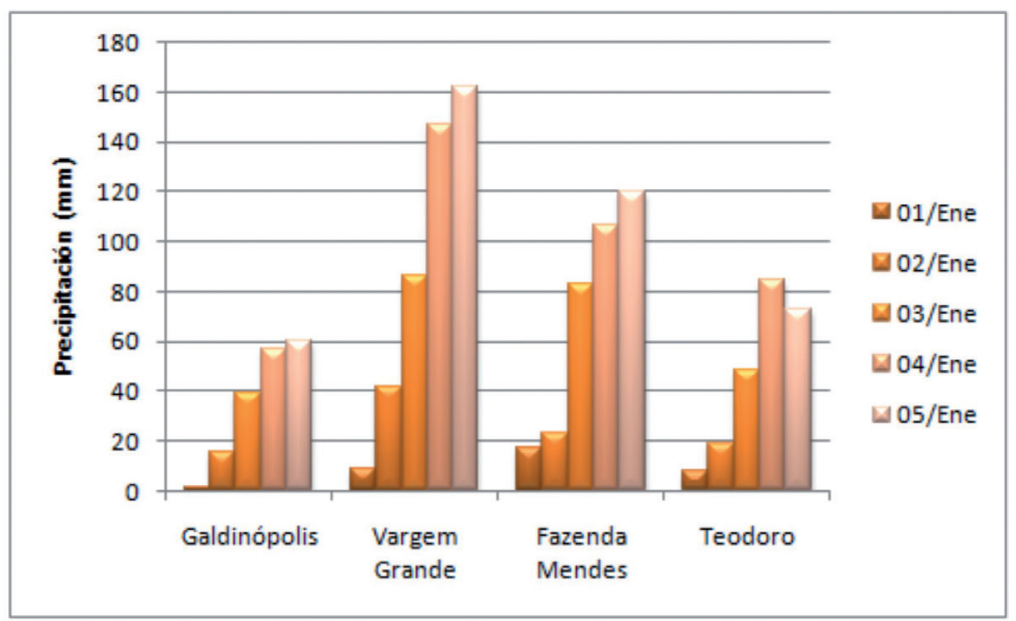

b)

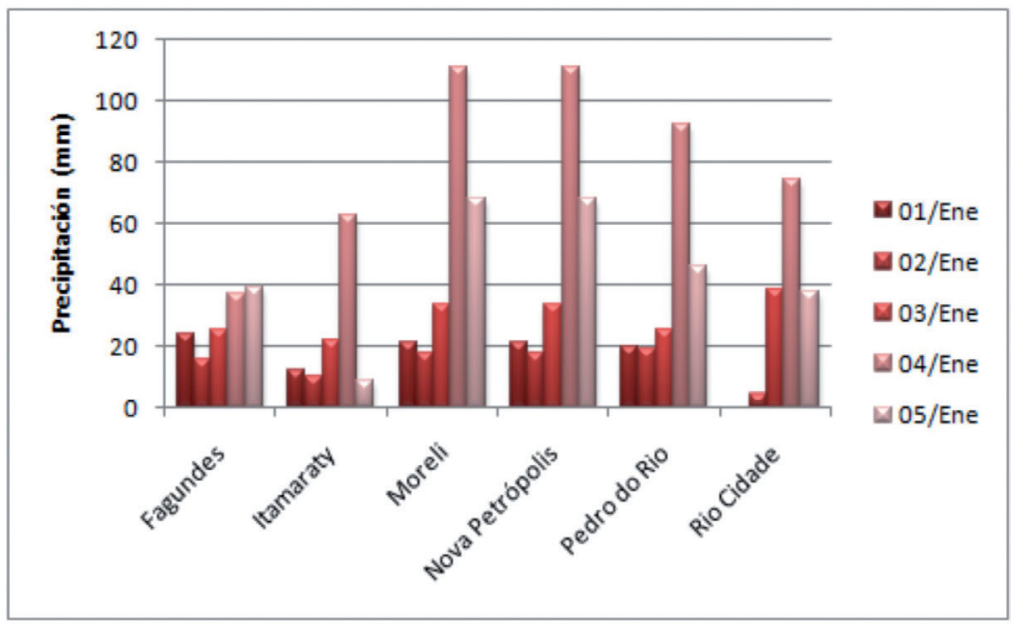

c)

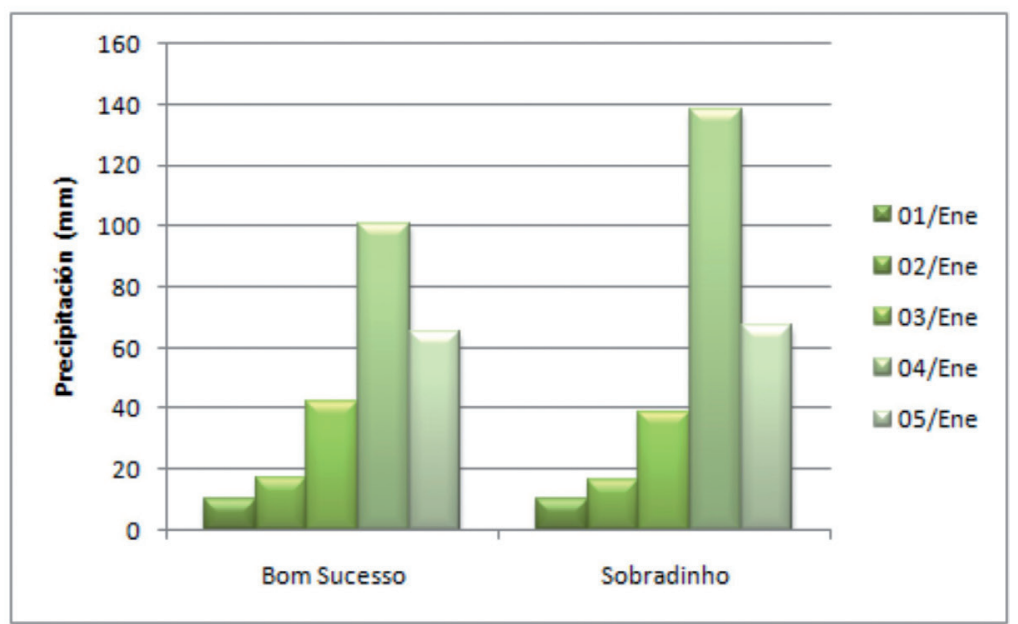

Figura 9. Precipitación diaria en el período 01-05 de Enero de 2007 en las estaciones meteorológicas de Nova Friburgo (a), Petrópolis (b) e Teresópolis (c).

Fig. 9. Daily precipitation in the period 01-05 January 2007 in the meteorological stations of Nova Friburgo (a), Petrópolis (b) and Teresópolis (c). 
Tabla1. Desastres notificados en las ciudades de Nova Friburgo, Petrópolis y Teresópolis en la década de 2000. Fuente: Secretaria Nacional de Defesa Civil. Las fechas en rojo indican PRECipitaciones SObre 100 mm (24h). SE* - Situación de EMERgencia.

Table1. Disasters reported in the cities of Nova Friburgo, Petrópolis and Teresópolis in the 2000s. Source: Secretaria Nacional de Defesa Civil. Days in red indicates rainfall over 100 mm (24). SE * - Emergency Situation.

\begin{tabular}{|c|c|c|c|c|c|}
\hline Ciudad & Evento & $\begin{array}{c}\text { Situación } \\
\text { legal }\end{array}$ & Fecha & Afectados & $\begin{array}{c}\text { Víctimas } \\
\text { fatales }\end{array}$ \\
\hline \multirow{2}{*}{ Nova Friburgo } & Crecida & $\mathrm{SE}^{*}$ & $19 / 01 / 2005$ & & 1 \\
\hline & Inundación & SE & $04 / 01 / 2007$ & 3.700 & 11 \\
\hline \multirow{16}{*}{ Petrópolis } & Crecida & & $02 / 01 / 2001$ & & \\
\hline & Crecida & SE & $24 / 12 / 2001$ & & 38 \\
\hline & Crecida & SE & $11 / 01 / 2003$ & & 17 \\
\hline & Inundación & SE & $12 / 03 / 2003$ & & \\
\hline & Inundación/Deslizamiento & & $21 / 12 / 2004$ & & \\
\hline & Inundación/Deslizamiento & SE & $04 / 01 / 2007$ & 30.000 & 3 \\
\hline & Crecida/Deslizamiento & SE & $03 / 02 / 2008$ & 45.000 & 11 \\
\hline & Crecida/Deslizamiento & SE & $10 / 01 / 2009$ & & \\
\hline & Crecida/Deslizamiento & & $19 / 01 / 2009$ & 100 & 2 \\
\hline & Crecida/Deslizamiento & & 10/10/2009 & & 4 \\
\hline & Crecida/Deslizamiento & & $04 / 12 / 2009$ & & 4 \\
\hline & Crecida/Deslizamiento & SE & $07 / 12 / 2009$ & & \\
\hline & Deslizamiento & SE & $23 / 02 / 2010$ & & \\
\hline & Crecida/Deslizamiento & SE & $06 / 04 / 2010$ & 15.000 & \\
\hline & Crecida/Deslizamiento & SE & $23 / 02 / 2010$ & & \\
\hline & Crecida/Deslizamiento & & $07 / 12 / 2010$ & & \\
\hline \multirow{8}{*}{ Teresópolis } & Deslizamiento & SE & $23 / 12 / 2001$ & & 03 \\
\hline & Deslizamiento & & $18 / 12 / 2002$ & & 01 \\
\hline & Deslizamiento & & $21 / 12 / 2002$ & & 14 \\
\hline & Deslizamiento & & $29 / 11 / 2006$ & & 03 \\
\hline & Deslizamiento & SE & $04 / 01 / 2007$ & 100 & 02 \\
\hline & Deslizamiento & SE & $05 / 03 / 2007$ & & \\
\hline & Crecida & & $25 / 02 / 2010$ & 31 & \\
\hline & Crecida & & $21 / 11 / 2010$ & & \\
\hline
\end{tabular}

En la Región Serrana de Rio de Janeiro, así como en todo el SEB, $80 \%$ del total anual de lluvias se encuentran entre los meses de octubre y marzo (ALVES et al. 2005). Sin embargo, los eventos de lluvias intensas vinculados a la acción de la SACZ están asociados a condiciones potenciales para la ocurrencia de desastres. Eso convierte la región vulnerable a los eventos extremos de precipitación que, asociados a la ocupación no organizada del territorio proyecta un escenario de riesgo para las ciudades.
A modo de ejemplo, la mayor probabilidad de ocurrenciadedeslizamientos, tantolosnaturales como aquellos de inducción antrópica, ocurren con un histórico pluviométrico caracterizado por 3 o 4 días de lluvias continuas, culminando con un episodio de lluvia de gran intensidad. En esas condiciones los suelos de las vertientes alcanzan niveles críticos de saturación y percolación interna de agua (SANTOS 2008).

El evento de gran precipitación en Enero de 2007, con víctimas fatales en las tres 
ciudades, corrobora la situación descrita. Las Figuras 9a-c muestran la precipitación diaria entre el 1-5 de Enero de 2007 en las estaciones meteorológicas de Nova Friburgo, Petrópolis y Teresópolis, respectivamente. El día de la declaración de la Situación de Emergencia (4/01/07) correspondió a lo máximo del período en la mayoría de las estaciones y valores aún elevados siguieron hasta el día 05 de Enero en algunas de ellas (Tabla 1).

La incursión de Sistemas Frontales en la región, la acción de los Sistemas Convectivos de Mesoescala y eventos de El Niño/La Niña son efectivamente determinantes, pero la acción de la Zona de Convergencia del Atlántico Sur parece ser un importante sistema meteorológico responsable por los totales pluviométricos sobre el promedio, pudiendo ser influenciada en intensidad en el área de actuación por los sistemas anteriormente mencionados. Sin embargo, esos sistemas meteorológicos son altamente controlados por procesos de macroescala como, por ejemplo, la ubicación y la intensidad de las corrientes en altos niveles y los episodios de bloqueo (NUNES et al. 2009). Además, las características topográficas de la Serra do Mar también influyen en la formación e intensificación de células convectivas, que pueden ser fundamentales en las observaciones de grandes totales de precipitaciones (LIEBMANN et al. 2001).

Pese a lo observado en los resultados, que son consistentes con las simulaciones de escenarios climáticos del IPCC, que proyectan un aumento en la precipitación, no es posible afirmar que existe efectivamente una tendencia de aumento de la precipitación para el periodo 1971-2010, o afirmar que la elevación de la precipitación de la década de 2000 con relación al periodo anterior sea también una tendencia, ya que el periodo de análisis de datos es relativamente corto. Según el Primer Informe de Evaluación Nacional Sobre Cambios Climáticos
(Painel Brasileiro de Mudanças Climáticas 2012), la década de 1970 presentó una fuerte variación climática y señala también que el análisis de tendencias en series relativamente cortas de parámetros climáticos, no son concluyentes. La mayoría de las tendencias detectadas en las precipitaciones de Brasil pueden ser explicadas por cambios de fase en oscilaciones decadales/interdecadales asociadas, por ejemplo a la Oscilación Multidecadal del Atlántico (OMA), que se da en escalas largas de tiempo y, por lo tanto, no puede ser considerada evidencia de cambios climáticos.

Además, deben ser consideradas las variaciones de los datos de precipitación de una ciudad para otra, debido a las características geográficas y topográficas locales, y de la complejidad de la dinámica atmosférica regional, que está vinculada a fenómenos de diversas escalas. De esta forma, es necesario acumular más datos de una serie más larga para tener más evidencias sobre las tendencias en la precipitación de la Región Serrana de Rio de Janeiro y también verificar su consistencia con las proyecciones climáticas del IPCC, que de momento no es posible realizar.

Sin embargo, el Primer Informe de Evaluación Nacional Sobre Cambios Climáticos (Painel Brasileiro de Mudanças Climáticas 2012) presenta datos de re-análisis, desde 1948, que provee evidencias del aumento de temperatura en gran parte de las regiones en Brasil de $0,6^{\circ} \mathrm{C} /$ década $\left(\sim 2,5^{\circ} \mathrm{C} / 40\right.$ años). La elevación de la temperatura también fue observada sobre el Atlántico Tropical, sugiriendo que puede haber ocurrido cambios en el contraste océano-atmósfera (PEZZI \& SOUZA 2009). En la cuenca del Atlántico Sur, las aguas más cálidas que lo normal, entre $2^{\circ} \mathrm{C}$ y $3^{\circ} \mathrm{C}$, también pueden estar contribuyendo para la elevación del número de eventos de precipitaciones intensas en la región, una vez que esas anomalías positivas de la TSM, próximo a la costa este de Brasil, vienen aumentando y expandiéndose desde 
agosto de 2009 (LIMA 2010). No obstante, no hay estudios concluyentes sobre la relación entre dichas pluviosidades intensas en la Región Sudeste de Brasil, la acción de la SACZ y variaciones de la temperatura del Atlántico Sur.

\section{CONCLUSIÓN}

La Región Serrana de Rio de Janeiro es vulnerable a los eventos extremos de precipitación, debido a su topografía montañosa, a la elevada concentración demográfica y por estar sujeta a la acción de sistemas meteorológicos que pueden causar intensa precipitación.

Considerando que, aunque no se pueda afirmar fehacientemente la existencia de una tendencia de aumento de las precipitaciones en la Región Serrana de Rio de Janeiro, los resultados alcanzados para la región dan cuenta de una intensificación de las precipitaciones extremas observadas en los últimos 40 años, además de la elevación del volumen de esas precipitaciones.

Los eventos de precipitación extrema han ocurrido en mayor número e intensidad en los años 2000, al que parecen asociados con la actuación de la Zona de Convergencia del Atlántico Sur. Los antecedentes científicos disponibles y los resultados alcanzados por esta investigación no permiten concluir respecto de las relaciones existentes entre las precipitaciones intensas de la Región Sudeste de Brasil, la acción de la SACZ y las variaciones de temperatura del Atlántico Sur.

No obstante, considerando los escenarios proyectados de cambios climáticos, también se puede concluir que las ciudades serranas no están preparadas para las tendencias climáticas esperadas, ni tampoco para una mayor frecuencia de eventos de precipitaciones extremas observadas en las últimas décadas, lo que produce significativos impactos socioeconómicos, fruto de mayor vulnerabilidad y exposición de las poblaciones locales en conjunto con el proceso de urbanización desordenada.

\section{AGRADECIMIENTO}

Se agradece a CNPq por el apoyo mediante beca de Iniciación Científica.

\section{REFERENCIAS}

ALMEIDA, F.F.M. \& C.D.R. CARNEIRO, 1998. Origem e evolução da Serra do Mar. RBG, 28 (2): 135-150.

ALVES, L.M., J.A. MARENGO, J.R. CAMARGO \& C. CASTRO, 2005. Início da estação chuvosa na região Sudeste do Brasil: Parte 1 - Estudos observacionais. Rev Bras Meteorol, 20 (3): 385-394.

CARVALHO, L.M.V., C. JONES \& B. LIEBMANN, 2002. Extreme precipitation events in southern South America and large-scale convective patterns in the South Atlantic Convergence Zone. J Climate, 15 (17): 2377-2394.

CARVALHO, L.M.V., C. JONES \& B. LIEBMANN, 2004. The South Atlantic Convergence Zone: Intensisty, Form, Persistence, and Relationships with Intraseasonal to Interannual Activity and Extreme Rainfall. J Climate, 17: 88-108.

COELHO NETTO, A.L., A.S. AVELAR, A.M. SATO, M.A. DIAS, M.B. SCHLEE \& A.B. NEGREIROS, 2012. Vulnerabilidade em geossistemas montanhosos e desastres causados por deslizamentos na interface floresta-urbana: controles geológicos, geomorfológicos e geoecológicos. En: LACERDA, W.A. et al. (Orgs.). Desastres Naturais Susceptibilidade e Riscos, Mitigação e Prevenção, Gestão e Ações Emergenciais. COPPE/UFRJ, Rio de Janeiro: 63-80. 
DANTAS, M.E., E. SHINZATO, A.I.M. MEDINA, C.R. SILVA, J. PIMENTEL, J.F. LUMBRERAS, S.B. CALDERANO \& F.A. CARVALHO, 2005. Diagnóstico Geoambiental do Estado do Rio de Janeiro. En: Oficina Internacional de Ordenamento Territorial Mineiro - CYTED (Ciencia y Tecnologia para el Desarollo Cooperacion IberoAmericana), 1: 2528 abr. 2005. Rio de Janeiro, 2005. CDROM, Sessão 4, 35 p. http://www.cprm. gov.br/publique/cgi/cgilua.exe/sys/start. htm?infoid $=700 \&$ sid $=94$.

DEPARTAMENTO DE RECURSOS MINERAIS DO ESTADO DO RIO DE JANEIRO, 2010a. Relatório sobre o evento do Morro da Carioca e Enseada do Bananal em Angra dos Reis, Relatório Interno, Diretoria de Geologia.

DEPARTAMENTO DE RECURSOS MINERAIS DO ESTADO DO RIO DE JANEIRO, 2010b. Relatório sobre o evento do Morro do Bumba, Relatório Interno, Diretoria de Geologia.

DEPARTAMENTO DE RECURSOS MINERAIS DO ESTADO DO RIO DE JANEIRO, 2011. Megadesastre da Serra, Relatório Interno, Diretoria de Geologia.

GAN, M., L.R. RODRIGUES \& V.B. RAO., 2009. Monção na América do Sul. In: Cavalcanti, I.F.A., Ferreira, N.J., Justi, M.G.A., Silva Dias, M.A.F. (Orgs.). Tempo e Clima no Brasil. Oficina de Textos, São Paulo, 462 pp.

GRIMM, A.M., 2003. The El Nino impact on the summer monsoon in Brazil: Regional processes versus remote influences. J Climate 16: 263-280.

GRIMM, A.M. \& R.G. TEDESCHI, 2009. ENSO and extreme rainfall events in South America. J Climate, 22: 1589-1609.
GROISMAN, P., R. KNIGHT, D. EASTERLING, T. KARL, G. HEGERL \& V. RAZUVAEV, 2005. Trends in tense precipitation in the climate record. J Climate, 8: 1326-1350.

KODAMA, Y., 1992. Large-Scale Common Features of Subtropical Precipitation Zones (the Baiu Frontal Zones, the SPCZ, and the SACZ) Part I: Characteristics of Subtropical Frontal Zones. Journal of Meteorological Society of Japan, 70 (4): 813-835.

KOUSKY, V.E., 1988. Pentad Outgoing Longwave Radiation Climatology for the South American Sector. Rev Bras Meteorol, 3: 217-231.

LIEBMANN, B., C. JONES \& L.M.V. CARVALHO, 2001. Interannual variability of daily extreme precipitation events in the state of São Paulo, Brazil. J Climate, 14: 208-218.

LIMA, K.C., P. SATYAMURTI \& J.P.R. FERNANDEZ, 2010. Large-scale atmospheric conditions associated with heavy rainfall episodes in Southeast Brazil. Theor Appl Climatol, 101 (1-2): 121-135.

LIMA, K.C., 2010. Episódios de precipitação intensa no Sudeste do Brasil e a influência das anomalias de temperatura da superfície do mar e da topografia. Tese de Doutorado do Curso de Pós-Graduação em Meteorologia, São José dos Campos: INPE.

MARENGO, J.A., L.M. ALVES, M.C. VALVERDE, R.P. ROCHA \& R. LABORBE, 2007. Eventos extremos em cenários regionalizados de Clima no Brasil e América do Sul para o Século XXI: Projeções de clima usando três modelos regionais. Relatório $\mathrm{N}^{\circ} 5$. Ministério do Meio Ambiente, 77 pp. 
MARENGO, J.A., 2009. Impactos de extremos relacionados com o tempo e o clima. Impactos sociais e econômicos. Boletim do Grupo de Pesquisas em Mudanças Climáticas (GPMC), 8, Edição Especial, Instituto Nacional de Pesquisas Espaciais: 1-4.

MINISTÉRIO DO MEIO AMBIENTE (MMA), 2011. Área atingida pela tragédia das chuvas - Região Serrana do Rio de Janeiro. Relatório de Inspeção. Brasília, 85 pp.

NUNES, L.H., A.K. VICENTE \& D.H. CANDIDO, 2009. Clima da região sudeste do Brasil. En: Cavalcanti, I.F.A., Ferreira, N.J., Justi da, M.G.A., Silva Dias, M.A.F. (Orgs.). Tempo e Clima no Brasil. Oficina de Textos, São Paulo, 462 pp.

PAINEL BRASILEIRO DE MUDANÇAS CLIMÁTICAS, 2012. Primeiro Relatório de Avaliação Nacional. Volume 1: Bases Cientificas das Mudanças Climáticas, 197 pp.

PAINEL INTERGOVERNAMENTAL SOBRE MUDANÇAS CLIMÁTICAS, 2007. Mudança do Clima: A Base das Ciências Físicas. Contribuição do Grupo de Trabalho I ao Quarto Relatório de Avaliação do Painel Intergovernamental sobre Mudança do Clima. Sumário para os Formuladores de Políticas, 25 p.
PEZZI, L. \& R.B. SOUZA, 2009. Variabilidade de mesoescala e interação oceano-atmosfera no Atlântico Sudoeste. En: Cavalcanti, I.F.A., Ferreira, N.J., Silva, M.G.A.J., Silva Dias, M.A.F. (Orgs.). Tempo e Clima no Brasil. Oficina de Textos, São Paulo, 462 pp.

QUADRO, M.F.L., 1994. Estudo de episódios de Zonas de Convergência do Atlântico Sul (ZCAS) sobre a América do Sul. 1994. 94f. Dissertação (Mestrado em Meteorologia) - Instituto Nacional de Pesquisas Espaciais, São José dos Campos.

SANTOS, A.R., 2004. A Grande Barreira da Serra do Mar. Ed. Nome da Rosa, São Paulo, 122 pp.

SANTOS, A.R., 2008. Diálogos Geológicos. Ed. Nome da Rosa, São Paulo, 182 pp.

SANTOS, A.R., 2012. Enchentes e Deslizamentos: causas e soluções. Ed. PINI, São Paulo, 124 pp.

SELUCH, E.M. \& S.C. CHOU, 2009. Synoptic patterns associated with landslide events in the Serra do Mar, Brazil. Theor Appl Climatol, 98 (1-2): 67-77.

VIANELLO L.R. \& A.R. ALVES, 1991. Meteorologia básica e aplicações. Viçosa: Editora UFV. 446 pp. 